\begin{tabular}{|c|c|}
\hline Title & $\begin{array}{l}\text { Spectral Narrowing of UV-V isible A bsorption and Emission Spectra of A nthracene-Derivatives in } \beta \text {-Cyclodextrin } \\
\text { Nanocavity: Effects of Host/Guest Stoichiometry }\end{array}$ \\
\hline Author(s) & Sato, Shin-Ichiro; Y oshizumi, Y uuki; Kiba, Takayuki; Murayama, A kihiro; A be, Shigeaki \\
\hline Citation & $\begin{array}{l}\text { Molecular Cry stal s and Liquid Crystals, 568(1), 60-65 } \\
\text { https://doi.org/10.1080/15421406.2012.710176 }\end{array}$ \\
\hline Issue Date & 2012-09-27 \\
\hline Doc URL & http:/hdl.handle.net/2115/53356 \\
\hline Rights & $\begin{array}{l}\text { This is an A uthor's A ccepted Manuscript of an article published in Molecular Cry stals and Liquid Crystals, 568(1), } \\
\text { Special Issue: Proceedings of the KJF International Conference on Organic Material s for Electronics and Photonics } \\
\text { (KJF-ICOMEP 2011) Part III, pp.60-65, 27 Sep. 2012, copyright @ T aylor \& Francis, available online at: } \\
\text { http://www.tandfonline.com/10.1080/15421406.2012.710176. }\end{array}$ \\
\hline Type & article (author version) \\
\hline File Information & MCLC5681_60-65.pdf \\
\hline
\end{tabular}

Instructions for use 
Title: Spectral narrowing of UV-visible absorption and emission spectra of anthracene-derivatives in $\beta$-cyclodextrin nanocavity: Effects of host/guest stoichiometry

Authors: Shin-ichiro Sato ${ }^{1 *}$, Yuuki Yoshizumi ${ }^{1}$, Takayuki Kiba ${ }^{2}$, Shigeaki Abe ${ }^{3}$, Akihiro Murayama $^{2}$

${ }^{1}$ Graduate School of Engineering, Hokkaido University, Sapporo Japan

Manuscript submitted to : $\quad$ Molecular Crystals and Liquid Crystals

Type of manuscript :

Article

Section of Journal : $\quad$ Special issue KJF2011

Correspondence and Proof to : Shin-ichiro Sato

Graduate School of Engineering,

Hokkaido University, Sapporo 060-8628, Japan

s-sato@eng.hokudai.ac.jp

Tel/Fax. + +81-11-706-6607

Contents :

Text

$5 \quad$ Pages

Tables

1

Figures 


\title{
Spectral narrowing of UV-visible absorption and emission spectra of anthracene-derivatives in $\beta$-cyclodextrin nanocavity: Effects of host/guest stoichiometry
}

\author{
Shin-ichiro Sato ${ }^{1 *}$, Yuuki Yoshizumi ${ }^{1}$, Takayuki Kiba ${ }^{2}$, Shigeaki Abe ${ }^{3}$, Akihiro Murayama ${ }^{2}$ \\ ${ }^{1}$ Graduate School of Engineering, Hokkaido University, Sapporo Japan \\ ${ }^{2}$ Graduate School of Information Science and Technology, Hokkaido University, Sapporo Japan \\ ${ }^{3}$ Graduate School of Dental Medicine, hokkaido University, Sapporo Japan
}

\begin{abstract}
Cyclodextrins (CD) are cyclic oligosaccharides consisting of six or more D-glucopyranose units, the interior of which forms a hydrophobic cavity. In the present study, we employed the CD confinement effects as a tool to suppress an electronic decoherence of guest wavefunction by reducing intermolecular interactions between a guest molecule and the surrounding environment. Effects of host/guest stoichiometry on the decoherence suppresion were investigated by comparing native $\beta-\mathrm{CD}$ with trimethyl- $\beta-\mathrm{CD}$ that should form only a 1:1 complex. Both fluorecence and fluorecence-excitation spectra of inclusion complexes exhibited spectral narrowings only for the $\beta$-CD host, suggesting that the formation of barrel-type $\beta$-CD dimer plays a key role for the pronounced spectral narrowings.
\end{abstract}

*Correspondence to: S.-i. Sato (E-mail: s-sato@eng.hokudai.ac.jp)

Keywords: Cyclodextrin, Inclusion complex, Fluorescence, Spectral narrowing, Decoherence 


\section{Introduction}

Cyclodextrins (CD) are cyclic oligosaccharides consisting of six or more D-glucopyranose units, the interior of which forms a hydrophobic cavity $[1,2]$. CDs are well-known in their ability to form inclusion complexes with hydrophobic molecules in aqueous solution and a number of studies have been performed about photochemical properties of the CD complexes including fluorescent probes such as pyrene, anthracene, coumarin and their derivatives [3-15]. We have focused on this confinement effects by the CD nanocavity as a tool to reduce interaction between a guest molecule and the surrounding environment, and have studied about an electronic spectral narrowing of these CD complexes. In our previous study, we reported the moderation of electronic decoherence of perylene in $\gamma$-CD aqueous solution at room temperature, as measured by an optical-phase-controlled pulse-pair quantum interferometer, combined with spectral lineshape analysis [16]. Interactions between guest molecules and surrounding environment are the origin of the electronic decoherence. Therefore, understanding this process is essential for the realization of quantum technologies such as bond-selective chemistry and quantum computing [17-20]. The quantum interference measurements showed that the electronic spectral narrowing in CD is largely caused by the lengthening of decoherence of the guest. However, this decoherence lengthening by the $\mathrm{CD}$ encapsulations was observed only for the specific combination of the host and guest molecules, and there remains some questions for the mechanism of spectral narrowings. In the present study, we investigated the effects of the host/guest stoichiometry on the spectral narrowing of the fluorescence and fluorescence-excitation (absorption) spectra of 9,10-dichroloanthracene (DCA) and 9, 10-dimethylanthracene (DMA) guests / $\beta$-CD and trimethyl- $\beta$-CD (TMB) hosts complexes. In literatures, $\beta-\mathrm{CD}$ is known to form a 2:1 complex easily (Figure 1). In contrast, TMB hardly forms 2:1 complexes because the presence of methyl groups hinders the barrel-type dimer 
formation.

\section{Materials and methods}

$\beta-\mathrm{CD}$ and TMB was purchased from Kanto Kagaku and used without further purifications. DCA and DMA was purchased from Aldrich and Tokyo Kasei, respectively and used as received. Chemical structures of these compounds are summarized in Figure 2. Each host/guest stock solution was prepared by mixing a $50 \mu$ l guest ethanol (Kanto Kagaku) solution $\left(1 \times 10^{-4}\right.$ mol

$\left.\mathrm{dm}^{-3}\right)$ with aqueous host solutions $\left(1 \times 10^{-3} \sim 1 \times 10^{-2} \mathrm{~mol} \mathrm{dm}{ }^{-3}\right)$ followed by stirring over night. A Milli-Q water purification system (Millipore, Bedford, MA) was used for purification of water. Steady-state fluorescence and fluorescence-excitation spectra were measured with an F-4500 fluorescence spectrometer (Hitachi, Tokyo) at room temperature.

\section{Results and Discussion}

Figure 3 shows fluorescence-excitaion (absorption) and fluorescence spectra of DCA in the presence of $\beta-\mathrm{CD}$ and TMB in aqueous solutions. The absorption and fluorescence spectra both showed well-resolved vibrational structures separated by ca. $1400 \mathrm{~cm}^{-1}$, and were in good relation of the mirror images each other. Obviously, spectral width is narrower in DCA/ $\beta-C D$ than in DCA/TMB, and sub vibrational structure separated by ca. $300 \mathrm{~cm}^{-1}$ can be clearly confirmed for the 0-0 transitions in DCA/TMB inclusion complexes. The inset in Figure 3 is the expansion around the 0-0 transition. The 0-0 band positions of DCA / TMB is red-shifted from those of $\mathrm{DCA} / \beta-\mathrm{CD}$, suggesting that DCA is in more polar environment in TMB than in $\beta-\mathrm{CD}$. It should be also noted that the Stokes shift, that is defined by the energy difference between a couple of absorption and emission $0-0$ bands, was very small for $\mathrm{DCA} / \beta-\mathrm{CD}$ in comparison with DCA/TMB. Because Stokes shift reflects the free energy relaxation by the reorientation of polar solvent after photo-excitation of solute (guest), the very small Stokes shift of DCA/ $\beta$-CD strongly 
suggest that DCA is in very non-polar environment in $\beta$-CD.

To estimate the spectral narrowing quantitatively, the lineshapes of 0-0 band were fitted by assuming Voigt function $S_{V}(\omega)$ :

$$
S_{V}(\omega)=\int_{-\infty}^{\infty} d \omega^{\prime} S_{L}\left(\omega^{\prime}\right) S_{G}\left(\omega+\omega_{0}-\omega^{\prime}\right)
$$

where $S_{L}(\omega)$ and $S_{G}(\omega)$ is Lorentzian and Gaussian lineshape, respectively. The spectral widths and Stokes shifts of DCA and DMA inclusion complexes were summarized in Table 1. The trend very similar with the DCA complexes was observed for the DMA complexes. That is, Spectral narrowing was not observed for DMA/TMB but observed for DMA/ $\beta-C D$, and the Stokes shift was smaller in DMA/ $\beta$-CD than in DMA/TMB. The spectral widths of DCA/ $\beta-C D$ and DMA/ $\beta-C D$ were comparable each other, but the spectral widths of DMA/TMB were much larger than those of DCA/TMB.

In order to confirm that these difference in spectral narrowing and Stokes shift is originated from host/guest stoichiometry, we carried out fluorescence titration measurements. Fluorescence intensity changes have been widely used for the determination of formation constants for CD and guest molecules, although the fluorescence intensity is sometimes replaced by fluorescence intensity ratio such as I/III vibronic band ratio for pyrene [21-24]. Fluorescence intensity ratio $R$ is given by

$$
\frac{1}{R-R_{0}}=\frac{1}{K_{1}\left(R_{1}-R_{0}\right)[C D]_{0}}+\frac{1}{R_{1}-R_{0}}
$$

for the 1:1 complex formation. On the other hand, $R$ is given by

$$
\frac{1}{R-R_{0}}=\frac{1}{K_{12}\left(R_{2}-R_{0}\right)[C D]_{0}^{2}}+\frac{1}{R_{2}-R_{0}}
$$

for the 2:1 complex formation, where the parameters $R_{0}$ and $R_{1}\left(R_{2}\right)$ donate the limiting fluorescence ratios in the absence and presence of hosts, respectively. $\mathrm{R}$ is the measured ratio at a given host concentration $[\mathrm{CD}]_{0} . \quad K_{1}$ and $K_{12}$ are the association constants for the 1:1 and 2:1 
complexes, respectively. In Figure $4,\left(R-R_{0}\right)^{-1}$ was plotted against $[C D]_{0}^{-1}$ or $[C D]_{0}^{-2}$ for DCA $/ \beta-C D$ and DCA/TMB. Such double reciprocal plot has been known to be a useful tool for the determination of the stoichiometry of guest molecule and CD[21-24]. Obviously, Equation (2) for the 1:1 complex well reproduce the experimental results for DCA/TMB, while Equation (3) for the 2:1 complex well reproduce the results for DCA/ $\beta$-CD. Therefore, barrel-type dimer formation of TMBs is strictly hindered by the permethylation of hydroxyl groups, as expected.

\section{Conclusions}

Spectral narrowing of DCA and DMA guests by the encapsulation of $\beta$-CD and TMB were investigated by means of fluorescence lineshape analysis. Fluorescence and fluorescence-excitation spectra of DCA and DMA were narrowed only for the $\beta-C D$ encapsulation. On the basis of fluorescence titration, it was shown that $\beta$-CD forms 2:1 complex, such as $\mathrm{DCA} /(\beta-\mathrm{CD})_{2}$, while TMB forms only 1:1 complexes. Therefore, the formation of 2:1 complex is one of key factors for the effective spectral narrowings.

\section{Acknowledgements}

This work was supported by Grants-in-Aid for Scientific Research (No. 20550001).

\section{Notes and References}

[1] Gerhard, W. (1994), Angew. Chem., Int. Ed., 33, 803.

[2] Saenger, W., Jacob, J., Gessler, K., Steiner, T., Hoffmann, D., Sanbe, H., Koizumi, K., Smith, S.M., Takaha, T. (1998), Chem. Rev., 98, 1787.

[3] Nowakowska, M., Smoluch, M., Sendor, D. (2001), J. Incl. Phenom. Macrocycl. Chem., 40, 213.

[4] Al-Kindy, S.M.Z., Suliman, F.E.O., Al-Hamadi, A.A. (2001), Analytical Sciences, 17, 539. [5] Starukh, G.N., Smirnova, N.P., Eremenko, A.M., Chuiko, A.A., Abdel-Shafi, A.A., Worrall, D.R., Wilkinson, F. (2001), Theoretical and Experimental Chemistry, 37, 103. 
[6] Sen, P., Roy, D., Mondal, S.K., Sahu, K., Ghosh, S., Bhattacharyya, K. (2005), J. Phys. Chem. A, 109, 9716.

[7] Li Chunmao, M.J.Z.Y. (2005), Luminescence, 20, 261.

[8] Roy, D., Mondal, S.K., Sahu, K., Ghosh, S., Sen, P., Bhattacharyya, K. (2005), J. Phys. Chem. A, 109, 7359.

[9] Chakraborty, A., Seth, D., Chakrabarty, D., Sarkar, N. (2006), Spectrochimica Acta Part A: Molecular and Biomolecular Spectroscopy, 64, 801.

[10] Sato, S.i., Nakamura, T., Nitobe, S., Kiba, T., Hosokawa, K., Kasajima, T., Otsuka, I., Akimoto, S., Kakuchi, T. (2006), J. Phys. Chem. B, 110, 21444.

[11] Ghosh, S., Mondal, S.K., Sahu, K., Bhattacharyya, K. (2006), J. Phys. Chem. A, 110, 13139.

[12] Ozawa, R., Hashimoto, T., Yamauchi, A., Suzuki, I., Smith, B.D., Hayashita, T. (2008), Analytical Sciences, 24, 207.

[13] Han, C.P., Li, H.B. (2008), Chinese Chemical Letters, 19, 215.

[14] Barra, M.i., Agha, K.A. (1997), J. Photochem. Photobiol. A: Chem., 109, 293.

[15] Dey, J., Warner, I.M. (1997), J. Phys. Chem. A, 101, 4872.

[16] Kiba, T., Kasajima, T., Nishimura, Y., Sato, S.-i. (2008), ChemPhysChem, 9, 241.

[17] Gordon, R.J., Rice, S.A. (1997), Annu. Rev. Phys. Chem., 48, 601.

[18] Rabitz, H., de Vivie-Riedle, R., Motzkus, M., Kompa, K. (2000), Science, 288, 824.

[19] Dantus, M., Lozovoy, V.V. (2004), Chem. Rev., 104, 1813.

[20] de Vivie-Riedle, R., Troppmann, U. (2007), Chem. Rev., 107, 5082.

[21] Munoz de la Pena, A., Ndou, T., Zung, J.B., Warner, I.M. (1991), J. Phys. Chem., 95, 3330.

[22] Munoz de la Pena, A., Ndou, T.T., Zung, J.B., Greene, K.L., Live, D.H., Warner, I.M. (1991), J. Am. Chem. Soc., 113, 1572.

[23] Zung, J.B., Munoz de la Pena, A., Ndou, T.T., Warner, I.M. (1991), J. Phys. Chem., 95, 6701.

[24] Kusumoto, Y. (1987), Chem. Phys. Lett., 136, 535. 
Table 1 Summary of spectral widths of 0-0 bands and Stokes shifts for each inclusion complexes

\begin{tabular}{ccccc}
\hline complex & $r_{v} / \mathrm{cm}^{-1}$ & $r_{l} / \mathrm{cm}^{-1}$ & $r_{g} / \mathrm{cm}^{-1}$ & stokes shift $/ \mathrm{cm}^{-1}$ \\
\hline DCA/ $\beta$-CD & 286 & 207 & 151 & 15 \\
DCA/TMB & 390 & 282 & 206 & 43 \\
DMA/ $/$-CD & 301 & 217 & 159 & 85 \\
DMA/TMB & 459 & 331 & 242 & 226 \\
\hline
\end{tabular}

$\gamma_{\mathrm{v}}$ (Voigt), $\gamma_{1}$ (Lorentzian), and $\gamma_{\mathrm{g}}$ (Gaussian) were obtained by band fittings assuming Voigt line shape function. 

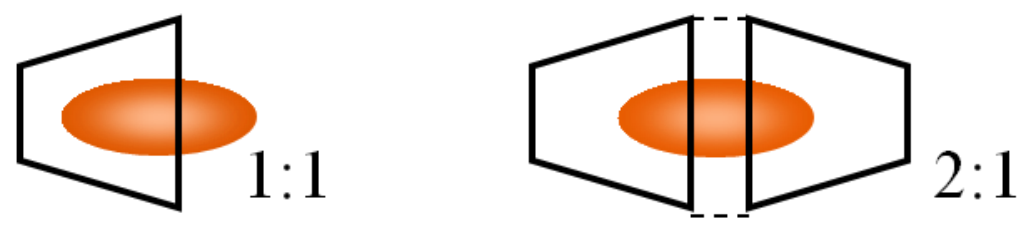

Figure 1 1:1 (left) and 2:1 (right) host/guest complexes 

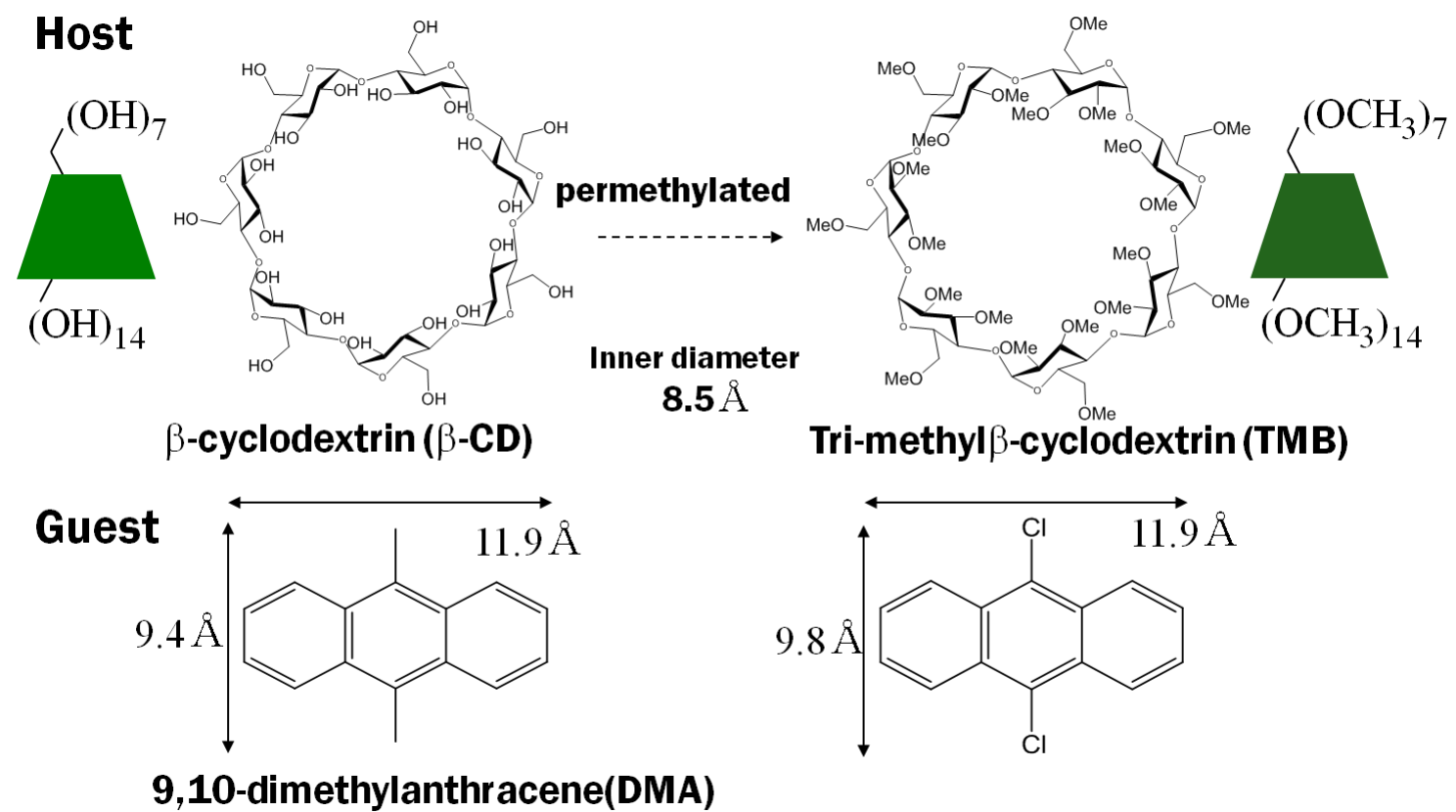

Figure 2 Chemical structures of $\beta$-CD (upper left), TMB (upper right), DMA (lower left), and DCA (lower right). 


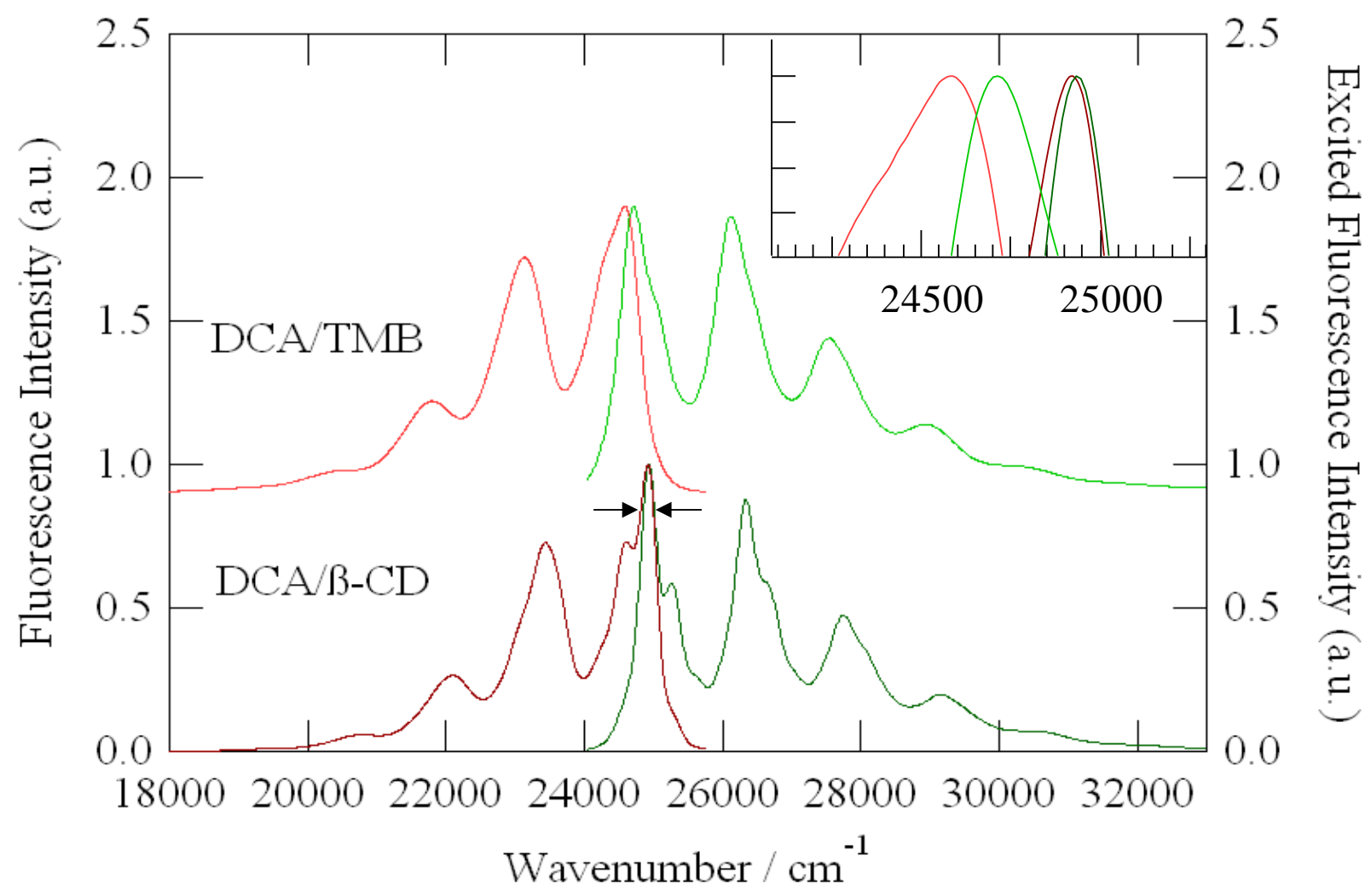

Figure 3 Fluorescence and Fluorescence-excitation spectra of the DCA/ $\beta$-CD and DCA / TMB inclusion complexes in aqueous solution in the presence of small amounts of EtOH. DCA concentration was $2.0 \times 10^{-6} \mathrm{M}$. 

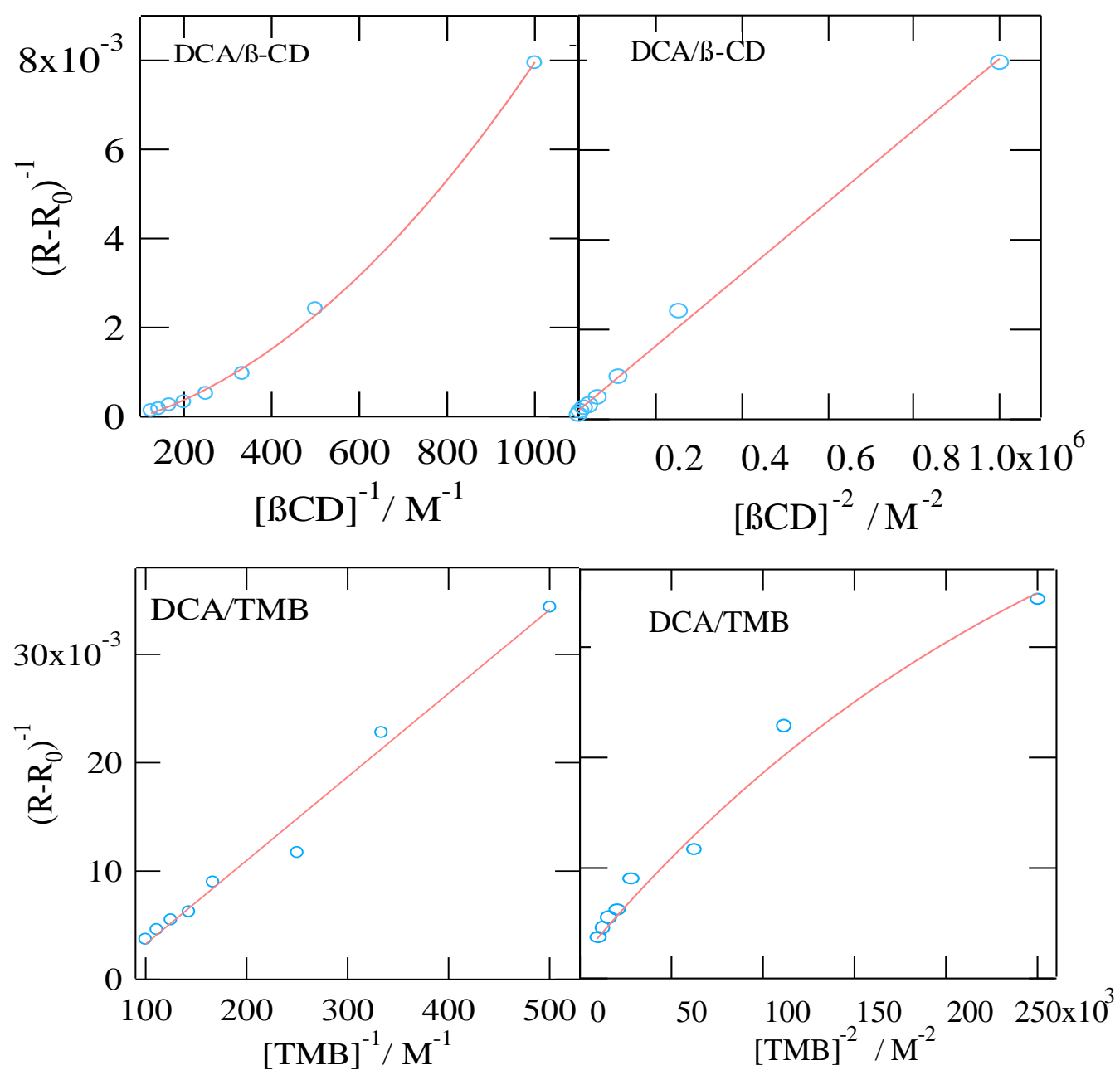

Figure 4 Reciprocal of fluorescence intensity vs reciprocal of concentrations (left) and reciprocal of squared concentrations (right) plots. The upper panel: DCA/ $\beta-C D$. The lower panel: DCA/TMB. 